\section{PSICANÁLISE E CONSTRUÇÃO DO CASO CLÍNICO: CONSIDERAÇÕES SOBRE UM DISPOSITIVO TERAPÊUTICO}

\author{
Psychoanalysis and Construction of the Clinical Case: \\ Considerations About a Therapeutic Device
}
Psicoanálisisy Construcción del Caso Clínico: Consideraciones Sobre un Dispositivo Terapéutico

\author{
Psychanalyse et Construction du Cas Clinique: Considérations \\ Concernant un Dispositif Thérapeutique
}

\section{Resumo}

Este artigo tem como objetivo discutir a construção do caso como um dispositivo clínico psicanalítico capaz de responder aos desafios do campo da saúde mental. A metodologia consistiu em um levantamento bibliográfico a partir de comentadores da psicanálise, tais como Vorcaro, Viganò e Figueiredo. A pesquisa teve como referência os descritores saúde mental, psicanálise e construção do caso clínico. De acordo com a discussão desses autores, a construção do caso clínico se caracteriza como uma proposta de trabalho e de investigação de orientação psicanalítica que norteia a conduta em equipe a partir da reflexão sobre as particularidades vivenciadas no contexto da saúde pública, da instituição e dos sintomas dos sujeitos, possibilitando a realização do trabalho em um solo comum a todos os profissionais da área da saúde mental. Tais características são consideradas fundamentais frente aos desafios do cenário da saúde mental que se caracteriza cada vez mais amplo e heterogêneo devido às mudanças advindas com a criação do SUS e da reforma psiquiátrica. Dessa forma, defendemos a construção do caso clínico como um dispositivo capaz de abranger a amplitude, as singularidades e as divergências, problematizando os pilares organizadores da clínica e os entraves institucionais, mobilizando o sujeito em seu diagnóstico permitindo que surja um espaço para que a subjetividade possa advir.

Palavras-chave: psicanálise; construção de caso clínico; dispositivo terapêutico; equipe; saúde mental.

\section{Abstract}

This article has as an objective to discuss the case construction as a psychoanalytical clinical device capable of providing an answer to the challenges of the mental health field. The methodology consists in a bibliographic mapping from psychoanalysis commentators that discuss clinical-institutional interventions in mental health, such as Vorcaro, Viganó and Figueiredo. The research had as reference the descriptors mental health, psychoanalysis and construction of clinical case. According to the discussion of said authors, the construction of the clinical case is characterized as a work and investigation proposal of psychoanalytical orientation that guides the team conduct from the reflection about the particularities experienced in the public health context, the institution and the subject's symptoms, enabling the accomplishment of the work in a soil common to all professionals of mental health area. The said characteristics are considered to be fundamental in facing the challenges of the mental health scenery which is characterized to be wider and heterogeneous by the time
Artigo Original

\section{Raquel Lima Pedrosa ${ }^{(1)}$ Leônia Cavalcante Teixeira ${ }^{(2)}$}

1) Profa. da Faculdade Integrada Tiradentes - Fits. Psicóloga graduada pela Universidade Federal de Alagoas. Mestre em psicologia pela Universidade de Fortaleza.

2) Profa. Titular do Programa de Pós - Graduação em Psicologia da Universidade de Fortaleza - UNIFOR. Bolsista de Produtividade em Pesquisa do CNPq. Dra. em Saúde Coletiva com Pós - Doutorado em Psicologia. Ms em Educação. Membro do GT da ANPEPP "Dispositivos clínicos em saúde mental"
Recebido em: 08/07/2013

Revisado em: 15/06/2015 Aceito em: 22/06/2015 
due to the changes resulted from the creation of the SUS and the psychiatric reform. This way, we defend the construction of the clinical case as a device able to cover the amplitude, the singularities and the divergences, raising problems about the organizer pillars of the clinic and the institutional hindrances, rallying the subject in his diagnostics allowing the emergence of a space for subjectivity to occur.

Keywords: psychoanalysis; clinical case construction; therapeutic device; Institution; mental health.

\section{Resumen}

Este artículo tiene como un objetivo discutir la construcción del caso clínico como un dispositivo clínico psicoanalítico capaz de responder a los desafíos del campo de la salud mental. La metodología consiste en un levantamiento bibliográfico a partir de comentaristas de la psicoanálisis que discuten intervenciones clínico-institucionales en salud mental, tales como Vorcaro, Viganó y Figueiredo. La investigación tuvo como referencia los descriptores salud mental, psicoanálisis y construcción del caso clínico. In acuerdo con la discusión de los autores, la construcción del caso clínico. De acuerdo con la discusión de los autores, la construcción del caso clínico se caracteriza como una propuesta de trabajo e investigación de orientación psicoanalitica que orienta la conducta in equipe a partir de la reflexión sobre las particularidades experimentadas en lo contexto de la salud pública, de la institución e los síntomas de los sujetos, posibilitando la realización del trabajo in suelo común a todos los profesionales de la área de la salud mental. Tales características san consideradas fundamentales frente a los desafios del escenario de la salud mental que se caracteriza cada vez más amplio y heterogéneo debido a las mudanzas advenidas con la creación del SUS y de la reforma psiquiátrica. Así, defendemos la construcción del caso clínico como un dispositivo capaz de alcanzar la amplitud, las singularidades y las divergencias, problematizando los pilares organizadores de la clínica y los obstáculos institucionales, movilizando el sujeto in suyo diagnostico permitiendo la insurgencia de un espacio para que la subjetividad posa advenir.

Palabras clave: psicoanálisis; construcción del caso clínico; dispositivo terapéutico; institución; salud mental.

\section{Résumé}

Ce travail a pour but discuter la construction du cas en tant que dispositif clinique psychanalytique capable de répondre aux défis du domaine de la santé mentale. La méthodologie repose sur une enquête bibliographique à partir de commentateurs de la psychalyse, tels que Vorcaro, Viganò et Figueiredo. La recherche a pour point de repère les descripteurs santé mentale, psychanalyse et construction du cas clinique. Selon la discussion de ces auteurs, la construction du cas clinique se caractérise par une proposition de travail et de recherche d'orientation psychanalytique qui guide la conduite en équipe à partir de la réflexion sur les particularités vécues dans le contexte de la santé publique, de l'institution et des symptômes des sujets possibilitant aussi la réalisation du travail sur un terrain commun à tous les professionnels du domaine de la santé mentale. Ces caractéristiques sont considérées fondamentales pour surmonter les défis du scénario de la santé mentale qui se trouve de plus en plus large et hétérogène, en raison des changements venus avec la mise en place du SUS et de la réforme psychiatrique. Ainsi, nous défendons la construction du cas clinique en tant que dispositif capable de toucher l'ampleur, les particularités et divergences, en problématisant les piliers organisateurs de la clinique et des entraves institutionnels et, qui, au même temps, mobilisera le sujet vers son diagnostic, permettant élever un espace pour que la subjectivité puisse se produire.

Mots-clés: psychanalyse; construction de cas clinique; dispositif thérapeutique; équipe; santé mentale.

Ao trabalharmos no campo da saúde mental nos deparamos com um universo amplo e heterogêneo, acompanhado de inúmeros impasses e desafios, sejam eles pelas demandas dos sujeitos implicados nos serviços de saúde mental, pelas limitações do trabalho em equipe, devido à formação profissional e às divergências teóricas e práticas do grupo, ou pelas fragilidades estruturais e políticas da rede de saúde pública.

A partir dos movimentos da Reforma Psiquiátrica, da instituição do Sistema Único de Saúde (SUS) pelas Leis Federais 8.080/1990 e 8.142/1990 e da ideia de clínica ampliada, proposta pelo governo a partir da Política Nacional de Humanização da Atenção e Gestão do SUS (Brasil, 2009), foram traçadas novas diretrizes, promovendo modificações nas instituições, na assistência e no processo de trabalho em saúde, assim como nos saberes, nas práticas, nos valores culturais e sociais.

A partir disso, foi estabelecida uma variedade de serviços que vão desde os programas comunitários, como o Programa de Saúde da Família (PSF), os Centros de Atenção Psicossocial (CAPS), passando pelos ambulatórios, onde se define uma proposta de reabilitação e ressocialização, visando diminuir, cada vez mais, a hegemonia do modelo hospitalocêntrico (Brasil, 2004).

Diante da amplitude e da heterogeneidade, Figueiredo $(2004,2005)$ defende que não é possível aspirar a uma homogeneização do campo de saúde mental, sob pena de reduzir a sua complexidade a uma visão simplista e mecânica. É preciso considerar a singularidade dos sujeitos, os possíveis dispositivos clínicos e a formação dos diversos profissionais, uma vez que o trabalho em equipe é central e determinante nos novos serviços de saúde mental.

Nesse contexto heterogêneo, é válido questionar: como 
constituir um solo comum de trabalho para os diferentes profissionais que atuam na área da saúde mental? Neste artigo, defendemos a construção do caso como uma das principais contribuições da psicanálise ao campo da saúde mental, ao trazê-lo como um possível dispositivo clínico capaz de responder aos desafios deste campo, considerando o trabalho em equipe e a singularidade dos pacientes.

É sabido que o contexto da saúde, de um modo geral, é permeado pela hegemonia do discurso biomédico. Nesta lógica, são produzidos sujeitos com identidades prontas. $\mathrm{Na}$ busca por uma cientificidade, cada vez mais, a particularidade dos sintomas é excluída, eles são considerados apenas para a identificação de um diagnóstico. Este último, por sua vez, refere-se a uma coleção de fenômenos que em nada diz respeito ao sujeito, mas que são cuidadosamente descritos e autorreferenciados, visto que o objetivo do diagnóstico é a eliminação do sintoma e não a busca pela sua origem ou causa na constituição do próprio sujeito.

Para Dor(1997), o diagnóstico, como prática biomédica, tem como objetivo determinar a natureza da doença, baseando-se em uma semiologia, e a classificação em um estado patológico, a partir de um quadro nosográfico. Nesse contexto, não se considera a maneira singular de interrogar o sofrimento psíquico do sujeito.

De acordo com Alkimim (2008), na perspectiva biomédica, uma vez estabelecido o diagnóstico, não há necessidade de escutar o sujeito. Este, por si só, já indica a conduta, o medicamento a ser utilizado e seus complementos, tais como a psicoterapia, a atenção social, entre outros. Nesse sentido, são valorizadas as histórias da doença, suas descrições e evolução, produzindo uma clínica das doenças e não do sujeito.

Cabe ressaltar que não se podem desprezar os avanços da biomedicina, nem a classificação e a nomenclatura dada pelos manuais de psiquiatria, porém a descrição, a nomeação e a diferenciação dos sintomas, agrupados nas ditas patologias, não devem ser reduzidos à mera classificação e à aplicação apriorística de condutas. Prova disso é a ineficácia observada no uso dos instrumentos habituais no tratamento da saúde mental, pautados no binômio medicação/resolução do sintoma, no qual ainda faltam palavras e sobram atos.

O trabalho em saúde mental deve ir além da resolução pela medicação, pela classificação diagnóstica e simples transcrições em prontuários. Este trabalho exige a problematização do que é diagnosticado, transcrito, implicando ainda a relação entre o profissional e o paciente, evidenciando o que escapa às classificações e às transcrições, por meio do esclarecimento de sua função e a posição de quem lê os dados (Vorcaro, 2010).

Para Alkimim (2008), diante do atual contexto da saúde mental e das (novas) exigências do campo, a apresentação social do sintoma mudou e, com ela, a necessidade de modificar o trabalho clínico. A ação do psicanalista, por exemplo, não pode se restringir à oferta de um acompanhamento terapêutico e a mera descrição de suas sessões. Segundo Delorenzo, Mezan e Cesarotto (2000), o trabalho do psicanalista deve vir acompanhado de comentários, interrogações, algo que faça daqueles sintomas que o sujeito apresenta algo também inusitado e enigmático, implicando a equipe e a instituição.

Ao abrir o espaço para o discurso analítico, algo da lógica institucional se subverte e a forma de compreender o sujeito, em crise, muda. A introdução desse discurso se caracteriza como uma estratégia de intervenção clínica na própria instituição. A partir do discurso analítico, são introduzidas as particularidades sintomáticas do sujeito no universo da instituição, dando brechas para a utilização do método da construção de caso clínico como dispositivo terapêutico (Bursztyn; Figueiredo, 2012).

Bursztyn e Figueiredo (2012) defendem a construção do caso clínico como uma proposta de trabalho e de investigação de orientação psicanalítica, ao privilegiar a discussão clínica, visa nortear a conduta em equipe a partir da transmissão da singularidade dos sintomas do sujeito.

Mais do que nortear uma equipe, a construção do caso clínico é defendida como um dispositivo terapêutico que se constitui como uma possibilidade de trabalho e investigação em um solo comum a todos os profissionais da área de saúde mental. Ela sustenta o trabalho em equipe pela lógica da singularidade do sujeito, caracterizando-se como uma das principais contribuições da psicanálise à saúde e ao trabalho institucional.

\section{A construção do caso clínico como dispositivo terapêutico psicanalítico: Apontamentos sobre questões teóricas e práticas}

A construção do caso clínico em saúde mental tem como objetivo a reflexão sobre as particularidades vivenciadas no contexto da saúde pública, da instituição e, particularmente, dos casos, uma vez em que esta tem a função de problematizar a generalização necessária à teoria, destacando a singularidade dos casos. Em outras palavras, construir um caso clínico é também escrever sobre a clínica. Assim o caso não é sinônimo do sujeito, mas sim uma construção, com base nos elementos que são reconhecidos em seu discurso, em uma situação terapêutica, e que permitem inferir sua posição subjetiva (Figueiredo, 2004; Vorcaro, 2010).

Nesse contexto, Viganò (1999) afirma também que construir um caso clínico em saúde mental é trabalhar no tempo de compreender. Por isso, é necessário estar atento aos movimentos, palavras e repetições do paciente que tragam questões em sua relação com a instituição e seus profissionais. Para tanto, ainda é necessário suportar o vazio do saber já difundido na saúde, um vazio que acolha a palavra do paciente e que o oriente na condução do caso. 
A construção do caso clínico não deve se limitar a descrições, por mais completas que sejam. Um relato compilado de acontecimentos e procedimentos nada mais é do que a anamnese. Esta seria a principal diferença da construção do caso defendida pelos autores que discutem o tema.

Para autores como Bursztyn e Figueiredo (2012), Viganò (1999) e Vorcaro (2010) a construção do caso clínico deve trazer, à cena do tratamento, o sujeito do inconsciente e suas produções, o qual só aparece pela via do discurso. É essa articulação que muda o eixo da discussão diagnóstica e do tratamento. Os autores defendem ainda que a construção do caso clínico também não coincide com a interpretação. "A construção é um arranjo dos elementos do discurso visando a uma conduta; a interpretação é pontual visando a um sentido" (Figueiredo, 2004, p.78).

Compreende-se construção deve ir ao encontro da partilha de elementos do caso que contribuam para um trabalho em conjunto, o que não seria possível pela via da interpretação. Surge, então, como uma possibilidade de indicar a direção do tratamento e como uma ação que favoreça a acolhida de fala e produção de sentidos e de singularidades.

Para melhor compreensão e aprofundamento desse dispositivo terapêutico, discutiremos o que Figueiredo (2004) denominou de "binômios da construção do caso", na tentativa de nos indicar os balizadores para a construção do caso e a diferenciação com os demais conceitos que podem vir a confundir o trabalho com este dispositivo clínico.

$\mathrm{O}$ primeiro binômio: história e caso. A história seria o relato clínico. É descrita pela autora como rica em detalhes, cenas e conteúdos, podendo assim ser fatigante; já o caso é o produto extraído das intervenções do analista durante o tratamento e do que é destacado do seu relato, ou seja, dos significantes do sujeito e suas produções a partir do processo analítico, incluindo as respostas do próprio analista (Figueiredo, 2004).

Não serão os inúmeros detalhes, nem a obediência a um determinado formato de escrita que formarão a riqueza do caso; tampouco o caso pode se reduzir a uma teorização formal, nem a um saber, puro e simples, sobre os problemas do paciente. A história do caso deve se fazer questão, para que se torne possível um trabalho, tanto na contemporaneidade do atendimento, quanto como fonte de pesquisas, o que ressalta o caráter fecundo do caso clínico como fonte de pesquisa nos campos do ensino e da transmissão da clínica/teoria/clínica.

Osegundo binômio: supervisão e construção. Figueiredo (2004) analisa o lugar da supervisão na construção do caso e lembra que uma supervisão psicanalítica não tem a intenção de decidir sobre a verdade dos casos atendidos. A construção está ligada à discussão e não se encerra ao término da sessão; ela continua e remete-se ao analista pesquisador que apresentou o caso.
Nikos e Moura (2000) comungam da ideia da autora acima e afirmam que, para que haja a construção do caso clínico, é preciso que a situação psicanalítica de supervisão sirva como espaço de interlocução entre o analista e a alteridade supervisora. As autoras ressaltam ainda que o principal instrumento dessa supervisão é a transferência.

Destacamos que a transferência é um aspecto fundamental que não se limita à relação analista e paciente, também está presente entre os pares e entre os profissionais e o próprio supervisor. É desse lugar e no manejo da transferência que o supervisor faz acontecer o trabalho em equipe.

Por fim, o terceiro binômio: conceitos e distinções. De acordo com Figueiredo (2004), a psicanálise é o efeito do encontro entre as ferramentas conceituais do analista e as contingências da história do sujeito. O que está em jogo não é um saber pronto e acabado; a teoria psicanalítica deve ser questionada durante todo o processo analítico, produzindo novos saberes.

Vale ainda uma última ressalva: a diferença entre a construção do caso e o estudo de caso. Ambas vêm se revelando além de um forte instrumento de formação, também um modo de avaliar e, portanto, de melhorar a qualidade clínica do trabalho. O psicanalista pode se utilizar da construção do caso clínico e do estudo de caso como meios de confrontar e interrogar, porém tais métodos são, muitas vezes, confundidos entre si, mas possuem epistemologias diferenciadas e produzem transmissões distintas do conhecimento psicanalítico.

De acordo com Nikos e Moura (2000) e Pereira, Godoy e Terçariol (2008), o estudo de caso é um procedimento utilizado habitualmente na clínica com o objetivo de compreender e planejar a intervenção, destacando-se pela possibilidade de integração de diferentes técnicas e campos do conhecimento. É um meio de organizar e reunir dados numerosos, de maneira a preservar seu caráter unitário. Caracteriza-se ainda como um estudo aprofundado de um objeto, de modo a ampliar e detalhar o conhecimento sobre o objeto, sendo que este objeto deve fazer parte de um todo, além de ser relevante ao caso, para que possa permitir um julgamento ou propor uma intervenção de forma que oriente a reflexão sobre uma cena, evento ou situação.

Os autores citados acima defendem que o estudo de caso deve ser realizado com o objetivo de promover uma análise do contexto e dos processos envolvidos com o objeto de estudo e é particularmente útil para responder perguntas do tipo "como" e "por que", por possibilitarem um estudo detalhado do fenômeno. Contrapõe-se à fragmentação do conhecimento, orientando-se a partir da preservação da complexidade humana. Nesta perspectiva, entra em cena não apenas a explicação dos quadros patológicos, mas também os modos do sujeito de sentir, representar e conduzir sua vida no processo de adoecer.

Além disso, considera-se, nesse contexto, que o 
interesse do psicanalista deve ir ao encontro da relação fenômeno-contexto e, assim como a construção do caso clínico, não deve se limitar às descrições. O caso clínico deve ser orientado por hipóteses, apoiados em conceitos, e que a coleta de dados seja dirigida por um esquema teórico.

Para Nikos e Moura (2000), o estudo de caso é o resultado da comunicação de uma experiência, na qual o terapeuta escolhe uma situação de tratamento para desenvolver sua hipótese. Neste caso, há uma delimitação conceitual daquilo que servirá como objeto de investigação. Para tanto, inicialmente, é registrada a anamnese que irá ajudar a compor a história clínica e a evolução das sessões, nas quais serão selecionados fenômenos, temas ou questões norteadoras da investigação. $O$ relato tem como base a cronologia dos fatos clínicos da história cuja narrativa se dá gradativamente, de acordo com a evolução do tratamento. As informações registradas pelo pesquisador servirão de base para uma interpretação, a partir da teoria.

Em geral, o estudo de caso serviria então como parâmetro para discussão de uma teoria subjacente à técnica utilizada na condução dessa experiência de tratamento. Em outras palavras, para o terapeuta que se utiliza do estudo de caso, sua alteridade fundamental é a teoria, é com ela com quem ele irá dialogar. Esta servirá de suporte e referência para o desenvolvimento de suas hipóteses.

É possível constatar que suas funções principais são informar e ilustrar, uma vez que basta a teoria para informar se a dimensão empírica que a clínica apresenta está ou não confirmada. Evidencia-se aqui mais uma diferença entre a construção do caso e o estudo de caso. Ao contrário do segundo método, a teoria não se configura como alteridade fundamental para a construção do caso.

Viganò (2010) destaca que a construção do caso clínico consiste em um movimento dialético em que as partes se invertem: o paciente se coloca na posição de docente, ele produz também a teoria ao passo que, na rede social, os profissionais se colocam em posição discente. Enquanto que, no estudo de caso, utiliza-se essencialmente a teoria com as respectivas variações, de acordo com a área de estudo.

A construção do caso, para a psicanálise, funda-se na articulação entre a experiência e o saber, entre clínica e teoria. Trata-se do esforço de transformar em saber a experiência do sujeito por meio do seu discurso. Nesta experiência, sua história poderá se desdobrar em novos sentidos, revelando elementos transferenciais. Trata-se, portanto, de uma via metodológica essencial na elaboração da teoria em clínica (Ferreira, 2005).

Nota-se que, para construir um caso é necessário partir da posição de não saber, ou seja, uma posição de total ignorância porque só quando o paciente verbaliza sua história é que aprendemos os caminhos de sua subjetividade. A história do paciente e suas significações, só ele sabe, isso não se aprende na Universidade. Portanto, isso implica operar com o saber do paciente e não com um saber sobre o paciente. Significa também criar um vazio de saber.

Vale ressaltar que outra característica da construção de caso é que esta é sempre provisória, pois está sujeita aos limites do material que já emergiu ou foi revelado na análise. Além disso, toda narrativa psicanalítica é uma narrativa da narrativa do paciente, ou seja, é o resultado de uma organização secundária e subjetiva do pensamento do analista. Qualquer outra forma que ela seja utilizada dará um sentido precário ao encontro da clínica. A pessoa da qual se fala ficará inacessível (Delorenzo et al., 2000, Gomes,1999 e Queiroz, 2002 ).

Para Queiroz (2002), a distância entre a história do paciente e a construção que se obtêm disso faz do caso clínico escrito uma organização ficcional e traz como propriedades principais a fronteira entre a vivência e a especulação, a apresentação do inaudível e do não dito, a transmissão, reinvenção e o progresso da psicanálise, produzindo novas metáforas e redes de significação.

Em relação às variações, o estudo de caso varia de acordo com a área em que é utilizado. Nikos e Moura (2000) afirmam que, em relação à psicopatologia, o estudo de caso se presta a aumentar o campo da explicação dos processos psicopatológicos, assim como amplia a equação de causação da patologia e do comportamento do sujeito. Já em Psicanálise, os autores descrevem que o estudo de caso, desde Freud, instaura um estilo de relatar documentalmente a evolução dos pacientes e serve de base para a sua metapsicologia.

O que caracteriza a construção do caso em saúde mental é a participação de uma equipe heterogênea, ou seja, diferentes profissionais e a pluralidade de referências teóricas e técnicas. É sabido que o trabalho em equipe é complexo e a forma como se estrutura é decisiva para o destino do trabalho.

Figueiredo $(2004,2005)$ destaca duas lógicas de organização que podem ajudar a compreender o problema, são elas: a organização hierárquica e a igualitária. Organizando-se a partir da primeira, a tendência é a verticalização do poder e saber, a cristalização das funções, além da burocratização da clínica. A partir da segunda organização, o poder tende a se horizontalizar, as funções se misturam, podendo ser confundidas a ponto de perder a referência da clínica e imobilizar o trabalho em equipe.

$\mathrm{Na}$ prática, é possível observar uma mistura entre os dois modos de funcionamento, de acordo com as situações que emergem no cotidiano. De uma ou outra forma, Borges (2010) e Figueiredo $(2004,2005)$ apostam na "transferência de trabalho" para a efetivação da construção do caso clínico. Este termo permite que se dissolvam os efeitos narcísicos imaginários que ocorrem tanto na confusão dos papéis do modelo igualitário como na fixação no modelo hierárquico e deve ser norteada pelo fato de que há um objetivo comum às diferentes profissões, ou seja, a concepção da clínica 
pautada no sujeito.

Para tanto, Borges (2010) cita determinadas premissas, decorrentes da psicanálise, necessárias aos operadores da transferência, tais como: não se colocar no lugar do paciente; evitar a interpretação pela intersubjetividade e não querer o "bem" do paciente. Em outras palavras, de maneira geral, mesmo na instituição, os pressupostos de uma análise são necessários de serem seguidos.

Quanto à última premissa citada, Figueiredo (2004) complementa que o importante é seguir o estilo do paciente para então indagar o que é pertinente ao seu sintoma, assim como fazê-lo se implicar nesse contexto, colher, de suas produções os indicadores do próprio tratamento.

Nota-se uma postura diferente do modelo biomédico tradicional que luta pela promoção do bem-estar e do desejo de cura. Deve-se, nesse caso, cotidianamente, promover alívio do sofrimento e da angústia como um meio e não como um fim. Do contrário, vamos ao encontro da cronificação do sujeito e de uma reabilitação moral e pedagógica daqueles considerados deficitários de racionalidade e responsabilidade.

Nesse sentido, em vez de perguntar: "O que podemos fazer por ele, a pergunta deve ser feita de outro modo: o que ele pode fazer para sair de tal ou tal situação com nosso suporte?" (Figueiredo, 2004, p. 81). Mudar o foco da pergunta significa suportar as ações do sujeito e chamá-lo à responsabilidade por seus atos, assim como significa mudar a lógica hierárquica dos saberes e funções da prática entre vários.

Essa é uma característica particular da "prática entre vários" defendida pela psicanálise (Borges, 2010; Figueiredo, 2004, 2005). A clínica psicanalítica constata que não é por servir ao bem comum que um sujeito se reconcilia com o seu próprio bem-estar. Pelo contrário, pode estar muito bem, mesmo em desacordo com as normas sociais de bem-estar, tal como no masoquismo, na repetição compulsiva, entre outros fatos que podem ser observados na prática clínica.

Há um saber a ser extraído do paciente que depende da transferência de trabalho, que pode ser estimulada não apenas pela presença do analista, mas, sobretudo, por meio do seu desejo, tomando a construção do caso como um meio de trabalho compartilhado. "É uma escolha contra a elisão do sujeito sob categorias universalizantes. É uma decisão pela elevação de suas respostas à dignidade de sinthomas" (Borges, 2010, p.6).

Esta forma de trabalho implica, de acordo com o que cada profissional da equipe esteja munido, um estilo pessoal de convívio, mais do que em saber prévio, universitário, como uma espécie de "desespecialização". Nesse sentido, Borges (2010) e Figueiredo (2004) defendem que o primeiro caso clínico deve ser o da própria instituição, que precisa constantemente rever seus protocolos e regulamentos hierarquizados e começar a se questionar sobre o que resta de fecundo na experiência diária, seja com os pacientes ou com a equipe.

A hipótese de Borges (2010, p. 7), para uma fórmula mínima da construção de caso no trabalho em equipe, seria: "quando um vazio de saber institucional suporta acolher um pedaço de real em ato, criam-se as condições de produção de um saber disjunto". Este último seria, portanto, aquele cuja assimilação pelo universal se revelou impossível e que só o dispositivo de uma construção do particular seria capaz de produzir.

Umúltimo elemento a ser discutido acerca da construção caso clínico diz respeito a sua elaboração propriamente dita, ou seja, os meios de suporte coletivo para a construção do caso. Este dispositivo está vinculado ao ato de escrever. Sabe-se que desde sua invenção, a narrativa é um hábito associado à psicanálise que, desde Freud, tem-se servido desse ato para a modelagem de conceitos e metáforas a partir do saber inconsciente que surge do discurso do analisante.

$\mathrm{Na}$ prática clínica, esse é o meio mais comum que comporta as mais diversas variações. Entre tantas, temos o relato contínuo do sujeito com o inconsciente; o modo da monografia exaustiva; o modo sessão por sessão e do tipo vinheta clínica, em que se busca recuperar um fragmento em destaque da história clínica.

Gomes (1999) e Queiroz (2002) discutem sobre as narrativas e afirmam que, do ponto de vista formal, o próprio exercício de escrever a clínica se cerca de normas, determinando estilos. Cita os achados, que vão desde os mais puros, ou seja, mais objetivos, descritivos e fiéis, ao que o analista entendeu do acontecimento clínico, no qual o narrador tenta manter a equidistância e indiferença pelos fatos que se desenrolam, até os estilos mais discursivos, em que há uma fala pessoal e subjetiva. Entre esses dois, há o romance psicológico, que é marcado por uma mistura de ambos.

A tensão narrativa na psicanálise entre uma escrita mais descritiva, mais objetiva, e outra mais discursiva, mais subjetiva, não é nova e embora procurem pensar a psicanálise por diferentes caminhos, é importante ressaltar que a necessidade da fundamentação metapsicológica, como ponto de ancoragem, permanece inquestionável em todas as formas de escrita (Gomes, 1999).

Vale ressaltar que, para a psicanálise, o caso representa mais que uma narrativa que supõe um estilo. De acordo com Queiroz (2002), independente do estilo em que se construa, o material comum a todos eles é o testemunho do encontro de cada sujeito com o seu pathos, registrado pela escuta do analista, produzindo uma terceira subjetividade ou uma "terceira organização ficcional" que adquire valor de verdade, como já discutido anteriormente.

Delorenzo et al. (2000, p. 108) apresentam o novo sujeito descrito como um produto misto: "misto do paciente real e de nós mesmos, naquilo que conhecemos dele (e que certamente é menos do que ele é em sua vida total), e também 
naquilo que ele se tornou ao ser integrado à vida psíquica do analista”. O paciente descrito na narrativa é, portanto, um personagem calcado sobre alguém efetivamente existente.

Embora o paciente e o trabalho específico realizado com ele sejam condições para a construção do caso, destacamos o elemento da subjetividade do analista, que aparece como fator estruturante do caso, uma vez que este também se refere aos processos internos do próprio narrador que se inquieta, persiste e persevera no desejo de saber, expondose enquanto escreve.

Em uma prática entre vários profissionais, como assim se configura para a construção de um caso, é sabido que a narrativa nem sempre é possível de ser realizada no dia a dia das clínicas e das instituições, limitando-se antes a uma conversação clínica. De acordo com Borges (2010), a conversação clínica trata de adaptar a cura ao caso, de retificar a posição dos operadores da transferência e de fazer a experiência clínica avançar.

O autor acredita que o inventário dos saberes disjuntos não precisa esperar necessariamente pela redação para se realizar, justificando que todo caso, de certa forma, é sempre urgente e a conversação clínica seria um recurso adaptado ao tempo da urgência. A conversação clínica também permitiria aos vários profissionais responder de forma imediata e precisa e não impediria o desenvolvimento posterior ou simultâneo de uma redação, na qual se exerça o exercício da problematização, evidenciando, sobretudo, o que escapa às relações, às classificações e as meras transcrições realizadas cotidianamente.

Para além dessa problematização, é possível afirmar que o desafio em questão visa também à reflexão sobre os pilares organizadores da clínica, em um cenário no qual inúmeros profissionais fazem parte de uma lógica que incita imobilizar o sujeito em seu diagnóstico e enfrentar os atravessamentos institucionais, deixando espaço para a subjetividade.

\section{Referências}

Alkimim, W. D. (2008). Construir o caso, a instituição enquanto exceção. CliniCAPS, 2(4), 1- 6 Revista eletrônica retirada do link http://pepsic.bvsalud.org/ pdf/clinic/v2n4/v2n4a07.pdf

Borges, A. (2010). Elementos mínimos para a construção do caso clínico na prática entre vários. CliniCAPS, 4(11), 1-7. Revista eletrônica retirada do link http:// www.clinicaps.com.br/clinicaps_pdf/Rev_13/ Padronizado\%20Cristiana\%20Ferreira.pdf

Brasil. (2004). Ministério da Saúde. Secretaria de Atenção à Saúde. DAPE. Coordenação Geral de Saúde Mental. Reforma psiquiátrica e política de saúde mental no
Brasil. Documento apresentado à Conferência Regional de Reforma dos Serviços de Saúde Mental: 15 anos depois de Caracas. Brasília: Editora do Ministério da Saúde.

Brasil. (2009). Ministério da Saúde. Secretaria de Atenção à Saúde. Política Nacional de Humanização da Atenção e Gestão do SUS. Clínica ampliada e compartilhada / Ministério da Saúde, Secretaria de Atenção à Saúde, Política Nacional de Humanização da Atenção e Gestão do SUS. Brasília: Ministério da Saúde.

Bursztyn, D.C \& Figueiredo, A. C. (2012). O tratamento do sintoma e a construção do caso na prática coletiva em saúde mental. Tempo psicanalítico. Rio de Janeiro, 44(1),131-145.

Delorenzo, R. M. T; Mezan, R.; Cesarotto, O. (2000). Narrar a clínica. Percurso, $25,2^{\circ} \mathrm{sem}$.

Dor, J. (1997) Estruturas e clínica psicanalitica. Rio de Janeiro: Taurus Editora.

Ferreira, A. P. (2005). A construção do caso clínico na internação psiquiátrica: uma direção para o plano terapêutico. Revista Latinoamericana de Psicopatologia Fundamental, 7 (2), 204-220.

Figueiredo, A. C. (2004). A construção do caso clínico: uma contribuição da psicanálise à psicopatologia e à saúde mental. Revista Latinoamericana de Psicopatologia Fundamental, 7 (1), 75-86.

Figueiredo, A. C. (2005). Uma proposta da psicanálise para o trabalho em equipe na atenção psicossocial. Mental. Barbacena, 3(5),44-55.

Gomes, P. B. (1999). Como se narra a psicanálise do final do milênio?. Percurso, 23(2), 63-72.

Nikos, I \& Moura, A. (2000). Estudo de caso, construção do caso e ensaio metapsicológico: da clínica psicanalítica à pesquisa psicanalítica. Pulsional, 13 (140/141), 6976.

Pereira, L. T. K.; Godoy, D. M. A; Terçariol, D. (2008). Estudo de caso como procedimento de pesquisa científica: reflexão a partir da clínica fonoaudióloga. Psicologia: Reflexão e crítica, 22(3), 422-29.

Queiroz, E. F. (2002) O estatuto do caso clínico. Revista Pulsional. São Paulo, 15(157), 33-40.

Viganò, C. (1999). A construção do caso clínico em saúde 
mental. Psicanálise e Saúde Mental Revista Curinga, Belo Horizonte -MG, 13, set, 50-59.

Viganò, C. (2010). A construção do caso clínico. Opção Lacaniana, 1(1), 1-9.

Vorcaro, A. (2010) Psicanálise e método científico: o lugar do caso clínico. In Pesquisa em psicanálise: uma transmissão na Universidade. Fuad Kyrillos Neto; Jacqueline Oliveira Moreira (Org.) Barbacena- MG: EdUEMG. 179p.

\section{Endereço para correspondência:}

Raquel Lima Pedrosa

Endereço: Rua Inácio Vasconcelos, $\mathrm{n}^{\circ}$ 263, ap. 1232,

Messejana. Fortaleza/Ceará. CEP: 60841-535.

Email: raquel.lpedrosa@gmail.com

\section{Endereço para correspondência:}

Leônia Cavalcante Teixeira

Endereço: Rua Henriqueta Galeno, no 1080-2301,

Dionísio Torres. Fortalezas/CE. CEP: 60135-240.

E-mail: leonia.ct@gmail.com 